\title{
Stages or phenotypes? A critical look at COVID-19 pathophysiology
}

\author{
Amit Jain *iD and D. John Doyle
}

(c) 2020 Springer-Verlag GmbH Germany, part of Springer Nature

\section{Dear Editor,}

We recently read with interest the editorial by Gattinoni et al. [1], conceptualizing 'type L' and 'type $\mathrm{H}^{\prime}$ (typical acute respiratory distress syndrome, ARDS) phenotypes for COVID-19. We hypothesize that what the authors label a type L phenotype is actually a typical Stage 2/Stage 3 of COVID-19 pneumonia [2]. Like any other pneumonia, COVID-19 pneumonia can result in complications like severe ARDS; we believe that referring to this as a 'type $\mathrm{H}$ phenotype' may lead to confusion. Furthermore, we are hesitant to accept the authors' hypothesis concerning the mechanism of hypoxia in the so-called type L phenotype. Here is our reasoning.

\section{Onset of hypoxia}

Gattinoni et al. proposed pulmonary vasoplegia as a key mechanism for the onset of severe hypoxia, but failed to give any plausible mechanism for this vasoplegia. We instead propose a 'epithelial-endothelial crosstalk' hypothesis that may provide a better explanation for the different stages of COVID-19 pneumonia:

i. Downregulation of ACE-2 on type II alveolar epithelial cells

SARS-CoV-2 enters the type II alveolar epithelial cells following binding of its spike protein to the ACE-2 receptor. This causes ACE-2 downregulation on the alveolar epithelium. Of note, SARS-CoV-2 seems to produce little or no direct cytopathic changes in the pulmonary epithelium [3].

*Correspondence: amitvasujain@gmail.com

Anesthesiology Institute, Cleveland Clinic Abu Dhabi, Al Maryah Island, Abu Dhabi, United Arab Emirates

\section{ii. Endothelial dysfunction}

The downregulation of ACE-2 on pulmonary epithelium results in unopposed effects of ACE-1 on the pulmonary capillary endothelial cells. Consequently, the level of protective ACE2-Ang 1-7-mas-R activity is reduced, while the level of harmful ACE 1-Ang II-AT1-R activity is increased. Possibly, the earliest deleterious effect of ACE 1-Ang II-AT1-R overactivity is the release of a potent vasoconstrictor, endothelin-1, from the pulmonary endothelium. ACE2-Ang 1-7-mas- $\mathrm{R}$ mediated constitutive endothelial nitric oxide (NO) synthase activation, and NO release is inhibited [4]. The intense pulmonary vasoconstriction so developed is severe, but uneven and results in the recruitment of capillary beds with relatively less vasoconstriction. As a result, the shunt fraction is increased and severe hypoxia ensues. As the disruption of the alveolar-capillary barrier progresses, proteins, fibrin, cells and fluid leak into the alveolar space, resulting in bilateral patchy ground glass opacities on CT scan.

\section{The evolution of the pulmonary manifestations of COVID-19 disease}

While patient-self-inflicted lung injury (P-SILI) is common, as a sole underlying mechanism for the progression of COVID-19 pneumonia to severe ARDS, it appears to be unlikely, especially, when early invasive mechanical ventilation policies are now largely adopted.

We hypothesize that 'endothelial-epithelial' interaction plays a major role in the disease progression: Following alveolar-capillary membrane disruption, SARS-CoV-2 enters the pulmonary capillaries and directly infects the pulmonary endothelial cells via ACE-2 protein on the luminal surfaces. As a result, endothelial cells assume a 'proinflammatory'/'procoagulant' phenotype. This accelerates Fas-induced apoptosis of alveolar epithelial and

\section{G Springer}


endothelial cells and orchestrates the cytokine storm and the progression of COVID-19 to ARDS [5].

\section{Implications for respiratory treatment}

The above hypothesis has treatment implications that warrant further investigation. It appears that high PEEP therapy, while preventing P-SILI, can increase shunt fraction; early intubation may not prevent a transition to ARDS, and early inhaled NO, steroids and anticoagulation therapy may be potentially helpful.

\section{Funding}

Support was provided solely from institutional and/or departmental sources.

\section{Compliance with ethical standards}

\section{Conflicts of interest}

The authors declare no competing interests.

\section{Publisher's Note}

Springer Nature remains neutral with regard to jurisdictional claims in published maps and institutional affiliations.

Accepted: 30 April 2020

Published online: 18 May 2020

References

1. Gattinoni L, Chiumello D, Caironi P et al (2020) COVID-19 pneumonia: different respiratory treatments for different phenotypes? Intensive Care Med. https://doi.org/10.1007/s00134-020-06033-2

2. Wang D, Hu B, Hu C et al (2020) Clinical characteristics of 138 hospitalized patients with 2019 novel coronavirus-infected pneumonia in Wuhan, China. JAMA. https://doi.org/10.1001/jama.2020.1585

3. Zhu N, Zhang D, Wang W, Li X, Yang B, Song J, Zhao X, Huang B, Shi W, Lu R, Niu P, Zhan F, Ma X, Wang D, Xu W, Wu G, Gao GF, Tan W, China Novel Coronavirus I, Research T (2020) A novel coronavirus from patients with pneumonia in China, 2019. N Engl J Med 382:727-733

4. Guignabert C, de Man F, Lombès M (2018) ACE2 as therapy for pulmonary arterial hypertension: the good outweighs the bad. Eur Respir J. https:// doi.org/10.1183/13993003.00848-2018

5. Millar FR, Summers C, Griffiths MJ, Toshner MR, Proudfoot AG (2006) The pulmonary endothelium in acute respiratory distress syndrome: insights and therapeutic opportunities. Thorax 71:462-473 